\title{
Thymosin $\beta 4$ alleviates renal fibrosis and tubular cell apoptosis through TGF- $\beta$ pathway inhibition in UUO rat models
}

Jing Yuan, Yan Shen, Xia Yang, Ying Xie, Xin Lin, Wen Zeng, Yingting Zhao, Maolu Tian and Yan Zha*

\begin{abstract}
Background: Thymosin $\beta 4$ (T/4) is closely associated with the cytoskeleton, inflammation, wound healing, angiogenesis, apoptosis, and myocardial regeneration, but the effects of T $\beta 4$ treatment on chronic renal tubular interstitial fibrosis (CRTIF) are poorly known. This study aimed to examine the effects of T $\beta 4$ on the renal apoptosis and the expression of transforming growth factor (TGF- $\beta$ ), E-cadherin, and a-smooth muscle actin ( $\alpha-S M A)$ in CRTIF rat models.

Methods: Male SD rats were randomized into four groups (sham group, unilateral ureteral obstruction (UUO) group, UUO + low-dose Tß4 group, and UUO + high-dose Tß4 group). The pathological changes of kidney tissue and its function were assessed two weeks after UUO. In renal interstitial tissue,TGF- $\beta$, E-cadherin and a-SMA expression was detected by western blot. In tubular epithelial cells, E-cadherin and a-SMA expression was detected using Real-time GPCR and western blot. Cell apoptosis of rat renal interstitial tissue and tubular epithelial cells was evaluated by immunofluorescence and western blot.

Results: Two weeks after UUO, no differences in blood urea nitrogen and creatinine were observed between the four groups $(P>0.05)$. Compared to the UUO group, TR4 treatment decreased the 24 -h proteinuria $(P<0.001)$ and reduced the area of pathological change $(P<0.01)$; this effect was more apparent in the UUO + high-dose Tß4 group. Compared to the UUO group, a significant decrease in TGF- $\beta$ and a-SMA protein expression was observed in the high-dose T $\beta 4$ group. The level of E-cadherin protein was lower in the UUO group than the T $\beta 4$ groups, and high-dose T $\beta 4$ treatment further increased E-cadherin expression and improved cell apoptosis in the renal interstitial tissue. Analysis of in vitro tubular epithelial cells showed that a-SMA mRNA and protein expression decreased, while E-cadherin mRNA and protein expression increased by T $\beta 4$ treatment. Similarly, these changes were more significant in the UUO + high-dose T $\beta 4$ group. T $\beta 4$ treatment improved the apoptosis of In vitro tubular epithelial cells compared with pure TGF- $\beta$ stimulation, and equally, the decrease of apoptosis was more apparent in the TGF- $\beta$ + high-dose T $\beta 4$ group.
\end{abstract}

Conclusions: T $\beta 4$ treatment might alleviate the renal fibrosis and apoptosis of tubular epithelial cells through TGF- $\beta$ pathway inhibition in UUO rats with CRTIF.

Keywords: Thymosin $\beta 4$, Transforming growth factor $\beta$, Renal fibrosis, Cell apoptosis

\footnotetext{
*Correspondence: zhayan72@126.com; yuanjinger@126.com

Department of Nephrology Guizhou Provincial People's Hospital, Guiyang

550002 , China
} 


\section{Background}

Chronic kidney disease (CKD) is characterized by abnormalities of kidney structure or function that are present $>3$ months and has an impact on the health of the patient [1]. According to recent epidemiological data, the prevalence of CKD in China has reached 10.8\%, which means that approximately 119.5 million people currently suffer from CKD in China [2]. Kidney disease is often due to direct injury, yet in many cases, this initial insult will initiate fibrogenesis, especially when healing process was applied by regeneration inadequate [3]. Renal fibrosis is the main pathological change of many CKDs and its pathological features include accumulation of ECM an extension or atrophy of the renal tubule [4].

Transforming growth factor (TGF)- $\beta$ is widely regarded as a key cytokine promoting fibrosis [5]. TGF- $\beta$ induces the transformation of tubular epithelial cells and mesangial cells into fibroblast cells, downregulates Ecadherin expression, and upregulates $\alpha$-smooth muscle actin (SMA) expression [6]. TGF- $\beta$ can induce fibroblast cells to express $\alpha$-SMA, accelerating the shrinkage of fibrotic tissues and leading to ischemia and hypoxia of renal tissues [7]. Therefore, inhibiting the expression of fibrosis -related factors such as TGF- $\beta$ might be a way to control or even reverse renal fibrosis, but there is a lack of reliable and effective drugs.

Thymosin $\beta 4$ (T $\beta 4)$ might be a solution against renal fibrosis. In recent years, a large number of in vivo and in vitro experiments have demonstrated that $T \beta 4$ is a multifunctional protein and has important roles in tissue repair and regeneration $[8,9]$. Because of its effects of anti-apoptosis, angiogenesis, anti-inflammation, and promotion of cell migration, $\mathrm{T} \beta 4$ plays central roles in promoting wound healing, tissue regeneration, and angiogenesis [10, 11]. Previous studies examined the inhibitory effects of various anti-fibrosis drugs including $\mathrm{T} \beta 4$ on fibrosis -related factors in rats with CKD [12, 13 ], but rare relevant research about the inhibitory effect of T $\beta 4$ on TGF- $\beta$ has been performed.

Therefore, we hypothesized TGF- $\beta$ is inhibited by T $\beta 4$, leading to alleviate renal fibrosis and cell apoptosis. This study aimed to examine the effects of different doses of T $\beta 4$ on the expression of TGF- $\beta$, E-cadherin, $\alpha$-SMA and apoptosis-related factors in CRTIF rats (Additional file 1).

\section{Methods}

\section{Animals}

Sixty Sprague Dawley male rats weighing 180-220 g and aged 6-8 weeks of specific pathogen-free grade were provided by the Chongqing Animal Center (permit number scxk-2007-0005). Rats were housed five rats/ cage at $23 \pm 2{ }^{\circ} \mathrm{C}$ and humidity of $55 \pm 2 \%$. Food and water intake was recorded daily. The litter was replaced daily. The rats were randomized to the sham group
( $n=15)$, unilateral ureteral obstruction (UUO) group $(n=15)$, UUO + low-dose T $\beta 4$ (1 mg/kg•d) group $(n=15)$, and UUO + high-dose T $\beta 4(5 \mathrm{mg} / \mathrm{kg} \cdot \mathrm{d})$ group $(n=15)$. The UUO and sham groups were given equal volumes of normal saline for intragastric lavage.

The rat model of UUO is currently regarded as the best animal model of progressive chronic renal tubule interstitial fibrosis [14]. The surgery was performed as described in the literature [14]. The rats received an intraperitoneal injection of $10 \%$ chloral hydrate $(0.3 \mathrm{~mL} /$ $100 \mathrm{~g}$ ) and were placed laterally on the right side. Skin preparation and disinfection were performed on the left rib on the back. An incision was made $0.5 \mathrm{~cm}$ at the left rib on the back. The abdominal cavity was opened and the left kidney and ureter was located and isolated using blunt dissection. The left ureter was held up with a tissue clamp and nipped with a hemostat at the upper middle segment. After the two ends were ligated, the left ureter between the ligated sutures was cut and removed. The incision was closed by suture layer. In the sham group, the abdominal cavity was opened but no tissue was removed.

\section{Culture of tubular epithelial cells}

Tubular epithelial cells (NRK-52E) were obtained from Nan Fang Medical University and grown in DMEM medium (Promo Cell, Heidelberg, Germany), containing $5 \mathrm{mM}$ glucose and $10 \%$ heat-inactivated FCS at $37{ }^{\circ} \mathrm{C}$ in $5 \%$ CO2. Tubular epithelial cells were stimulated with $2.5 \mathrm{ng} / \mathrm{ml}$ TGF- $\beta$ for $72 \mathrm{~h}$ in the TGF- $\beta$ group, $1 \mu \mathrm{g} / \mathrm{ml} \mathrm{T} \beta 4$ and $5 \mu \mathrm{g} / \mathrm{ml} \mathrm{T} \beta 4$ in the low dose T $\beta 4$ group and high dose $\mathrm{T} \beta 4$ group for $48 \mathrm{~h}$ after TGF- $\beta$ treated cells, respectively.

\section{Sample collection}

Blood samples were taken from the carotid artery under anesthesia two weeks after UUO. The serum was separated by centrifugation and was used for detection of blood urea nitrogen (BUN) and creatinine. All rats were sacrificed by cervical dislocation, the left kidney was dissected, and the capsule was peeled off. The kidney was fixed in 10\% formaldehyde. Paraffin sections were prepared, routine hematoxylin and eosin (H\&E) and periodic acid-Schiff (PAS) staining were performed. The remaining sections were used for immunofluorescence. Remaining kidney tissues were stored at $-80{ }^{\circ} \mathrm{C}$.

\section{Kidney histology and morphology}

Paraffin sections were processed for PAS staining. Changes of the renal tubule were observed in a doubleblind manner under a light microscope $[15,16]$. Three sections were chosen from each rat and ten fields of the renal interstitial tissue (avoiding the glomerulus and large blood vessels) were randomly chosen from each section. The area of the renal interstitial tissue was measured by Image pro-Plus6.0 under 200xmagnification. The 
area ratio of fibrosis and total interstitial tissue was measured in each field. The mean value was used for analysis.

\section{Real-time qPCR}

Total RNA was prepared from the tubular epithelial cells using RNAEasy minikit (Invitrogen, Carlsbad, CA) and reverse transcribed according to the manufacturer's instructions. Primers for E-cadherin, $\alpha$-SMA and $\beta$-actin were designed and synthesized based on published sequences of these genes as listed in Table 1. Real-time qPCR was performed using SYBR Green PCR Master Mix (Toyobo, Osaka, Japan) and a Rotor- Gene-3000A Real-time PCR System (Corbett, Sydney, Australia) according to the manufacturer's protocol. The target gene expression was normalized to $\alpha \beta$-actin mRNA expression and presented as fold-change compared to the control experiments.

\section{Western blot}

Kidney tissues were placed in radio-immunoprecipitation assay (RIPA) buffer on ice, fully mixed, incubated for $30 \mathrm{~min}$, and centrifuged at $12,000 \mathrm{rpm}$ at $4{ }^{\circ} \mathrm{C}$ for $20 \mathrm{~min}$. The supernatant was taken for protein measurement using the bicinchoninic acid assay (BCA) protein detection kit (Sigma, St Louis, Mo, USA). Sodium dodecyl sulfate(SDS) sample buffer (125 mM Tris- $\mathrm{HCl} \mathrm{pH} 6.8,4 \%$ SDS, $20 \%$ glycerol, $100 \mathrm{mM}$ dithiothreitol and $0.2 \%$ bromophenol blue) was added. The sample was heated at $100{ }^{\circ} \mathrm{C}$ for $5 \mathrm{~min}$ and stored at $-20{ }^{\circ} \mathrm{C}$.

Proteins $(50 \mu \mathrm{g})$ were separated using $8 \%$ or $10 \%$ SDSpolyacrylamide gels, and the proteins were transferred to nitrocellulose membranes (Amersham, GE Healthcare, Waukesha, WI, USA). The membranes were incubated with antibodies against E-cadherin (1:2000; GIBCO, Invitrogen Inc., Carlsbad, CA, USA), TGF- $\beta$ (1:3000 GIBCO, Invitrogen Inc., Carlsbad, CA, USA), $\alpha$-SMA (1:2000; GIBCO, Invitrogen Inc., Carlsbad, CA, USA),Cleaved Caspase 3 (1:1000, Abcam, UK), Bax(1:1000, Abcam, UK), and Bcl-2 antibodies (1:1500, Abcam, UK) in tris-buffered saline-tween 20 (TBST) containing $2 \%$ BSA overnight at $4{ }^{\circ} \mathrm{C}$. After washing, membranes were incubated with horseradish peroxidase-labeled secondary antibody (1:1000;GIBCO, Invitrogen Inc., Carlsbad, CA, USA) in TBST containing 2\% BSA at room temperature for $1 \mathrm{~h}$. Enhanced chemiluminescence (Amersham, GE
Healthcare, Waukesha, WI, USA) was used for detection. Signal intensity was analyzed using the ScionImage gel image analysis system (Scion Corporation, Frederick, MD, USA). $\beta$-actin was used as internal control. Quantitative analysis of the western blot bands was conducted using the Labwork analysis software. The 15 samples per group was detected.

\section{Apoptotic determination}

TUNEL staining was carried out using a promega apoptosis detection kit. Immunofluorescence for TUNEL staining was performed with Alexa Fluor 594-conjugated goat anti-mouse IgG (1:500; Invitrogen). The glass was mounted with cover slips containing Vectashield mounting medium with 4',6-diamidino-2-phenylindole (DAPI; Sigma) and imaged under an fluorescent microscope.

\section{Statistical analysis}

SPSS16.0 (IBM, Armonk, NY, USA) was used for statistical analysis. Continuous data are expressed as mean \pm standard deviation (SD) and were analyzed using one-way ANOVA followed by the LSD post hoc test. Two-sided $P$-values $<0.05$ were considered statistically significant. The data was analyzed in normally distributed by Kolmogorov-Smirnov $(P>0.05)$, data obeyed the normal distribution.

\section{Results}

Effects of T $\beta 4$ on kidney functions and area of renal interstitial fibrosis in the rat model of UUO

Proteinuria was reduced in the T $\beta 4$ groups $(P<0.01)$, and the decrease was more obvious in the UUO + highdose T $\beta 4$ group $(P<0.05)$. No difference was found in kidney function $(P>0.05)$, but pathological changes in the renal tubulointerstitium was significantly reduced $(P<0.05)$, particularly in the high-dose group (Table 2$)$.

\section{Effect of different doses of T $\beta 4$ on histology and morphology of kidney tissue in the rat model of UUO}

Normal morphology of kidney tissue is the base of kidney function. In the sham group, organization of kidney tissue was in good order and no abnormal pathological changes were observed in the glomerulus and renal tubule (Fig. 1a). In the UUO group, severe atrophy was seen in the glomerulus, the capsule area was diffusely widened, the

Table 1 Primer sequences, product size and annealing temperatures used in RT-PCR

\begin{tabular}{|c|c|c|c|c|}
\hline Gene & & & Product size $(\mathrm{pb})$ & Temperature $\left({ }^{\circ} \mathrm{C}\right.$ \\
\hline \multirow[t]{2}{*}{$\bar{\beta}$-actin } & Right & GTTGTCGACGACGAGCG & 93 & 60 \\
\hline & Left & GCACAGAGCCTCGCCTT & & 50 \\
\hline \multirow[t]{2}{*}{ E-cadherin } & Right & GACCGGTGCAATCTTCAAA & 93 & 59 \\
\hline & Left & TTGACGCCGAGAGCTACAC & & 60 \\
\hline \multirow[t]{2}{*}{ a-SMA } & Right & GCGTGATTTCCAGCACATAA & 101 & 59 \\
\hline & Left & ATACTTGACCGGGGTCATCC & & 60 \\
\hline
\end{tabular}


Table 2 Changes of kidney function and area ratio of renal interstitial fibrosis in different groups

\begin{tabular}{|c|c|c|c|c|}
\hline & Sham & UUO & UUO + low-dose Tß4 & UUO + high-dose Tß4 \\
\hline Proteinuria (mg/24 h) & $13.32 \pm 4.19$ & $251.30 \pm 28.63^{\Delta}$ & $101.31 \pm 19.04^{\#}$ & $69.25 \pm 10.27^{\mathbf{\Lambda}}$ \\
\hline BUN (mmol/L) & $6.31 \pm 1.02$ & $9.24 \pm 2.31$ & $7.82 \pm 1.95$ & $7.07 \pm 1.67$ \\
\hline Creatinine (mmol/L) & $50.35 \pm 3.78$ & $81.24 \pm 12.54$ & $69.82 \pm 13.68$ & $64.73 \pm 14.06$ \\
\hline Area ratio of fibrosis and total interstitial tissue & $0.053 \pm 0.008$ & $0.55 \pm 0.04^{\Delta}$ & $0.31 \pm 0.05^{\#}$ & $0.074 \pm 0.006^{*}$ \\
\hline
\end{tabular}

$\triangle P<0.001$ vs. the sham group

$\# P<0.01$ vs. the UUO group

$\Delta P<0.01,{ }^{*} P<0.05$ vs. the UUO + low-dose T $\beta 4$ group

UUO unilateral ureteral obstruction, $T \beta 4$ thymosin $\beta 4, B U N$ blood urea nitrogen

tubular cavity was enlarged, and the epithelial cells in the proximal convoluted tubule around the medullary loop showed vacuolar degeneration and were swollen. In the area with severe pathological changes, exfoliated epithelial cells were observed and the renal interstitial tissue was disrupted and atrophied, and had a tendency for fibrosis (Fig. 1b). Pathological damage was alleviated to a greater extent in the UUO + high-dose T $\beta 4$ group (Fig. 1d) compared to the UUO + low-dose T $\beta 4$ group (Fig. 1c).

\section{Detection of TGF- $\beta$ expression in the rat kidney tissue by western blot}

TGF- $\beta$ protein expression in the UUO group was markedly increased compared to the sham group (Fig. 2a-b; $P<0.01)$. The expression was significantly decreased in the low-dose T $\beta 4$ group compared to the model group (Fig. 2a-b) $(P<0.05)$, and the expression was even decreased to a greater extent in the high-dose $\mathrm{T} \beta 4$ group (Fig. 2a-b) $(P<0.05)$.

\section{Detection of E-cadherin and a-SMA expressions in kidney tissue from different groups by western blot}

E-cadherin expression in the UUO group was significantly lower compared to the sham group (Fig. 3a, c) $(P<0.01)$. The expression of E-cadherin in the $\mathrm{UUO}+$ low-dose group was markedly increased compared to the UUO group $(P<0.05)$. The increase in E-cadherin expression was greater in the high-dose group compared to the low-dose group (Fig. 3a, c) $(P<0.05) . \alpha$-SMA expression in the UUO group was
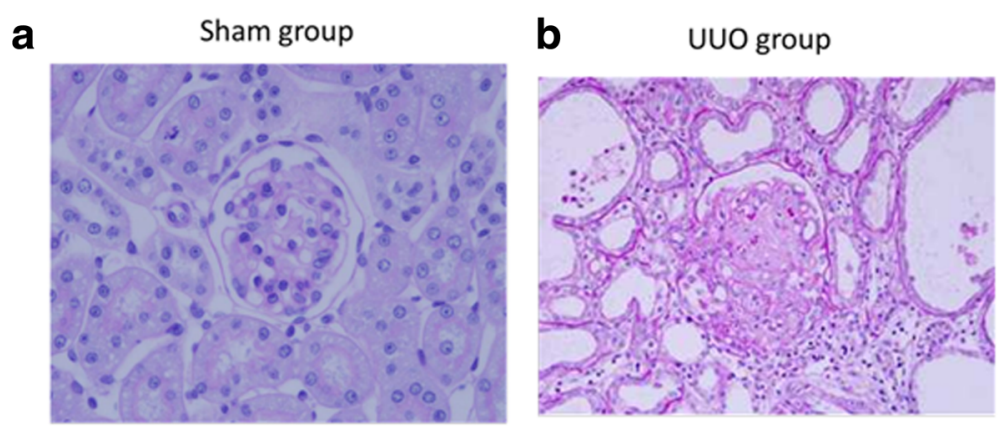

C low dose $\mathrm{T} \beta 4$ group

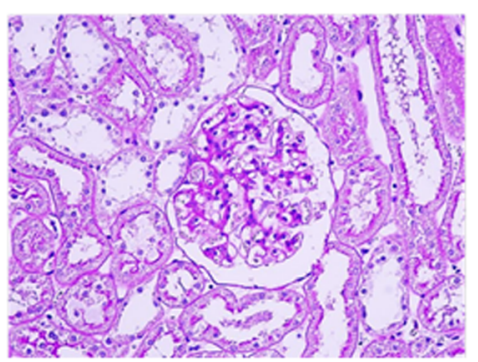

d high dose T $\beta 4$ group

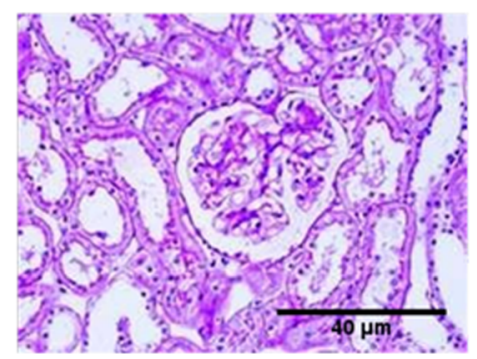

Fig. 1 Effects of different doses of TR4 on the pathological changes in kidney tissue in a rat model of UUO. a In the sham group, regular arrangement of tissue structures of the kidney with normal glomerulus and renal tubule. $\mathbf{b}$ In the UUO group, severe atrophy of the glomerulus, diffusely widened capsule area, enlarged tubular cavity, vacuolar degeneration, and swollen epithelial cells in the proximal convoluted tubule around the medullary loop, exfoliated epithelial cells in the area with severe pathological changes, disrupted and atrophy renal interstitial tissue, and fibrosis were observed. c The pathological damage was alleviated in the UUO + low-dose Tß4 group. $\mathbf{d}$ The damages were further alleviated in the UUO + high-dose Tß4 group. Periodic Acid-Schiff staining $\times 200$ magnification 


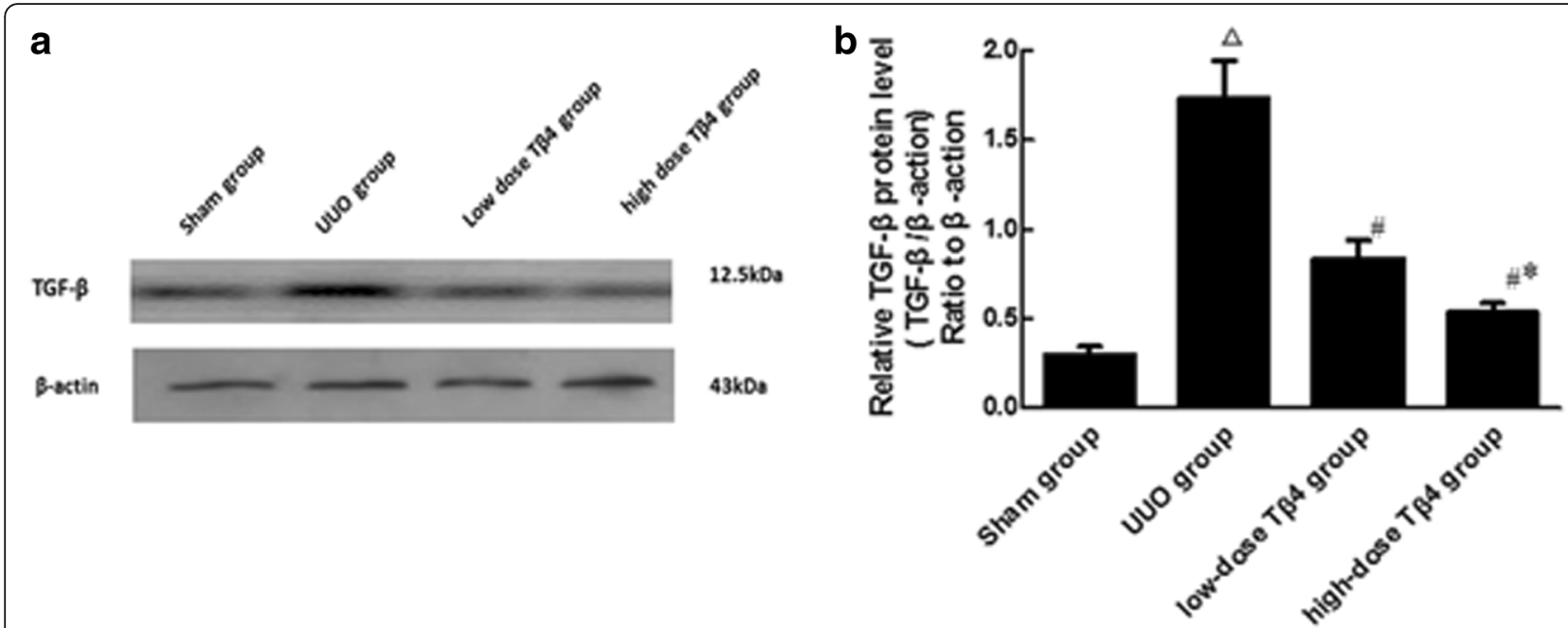

Fig. 2 Effects of different doses of T $\beta 4$ on TGF- $\beta$ expression in renal tubulointerstitial tissue of UUO rat model. a Western blot. b Quantification of A. ${ }^{\Delta} P<0.01$ vs. the sham group, ${ }^{\#} P<0.05$ vs. the UUO group, ${ }^{*} P<0.05$ vs. the UUO + low-dose T $\beta 4$ group

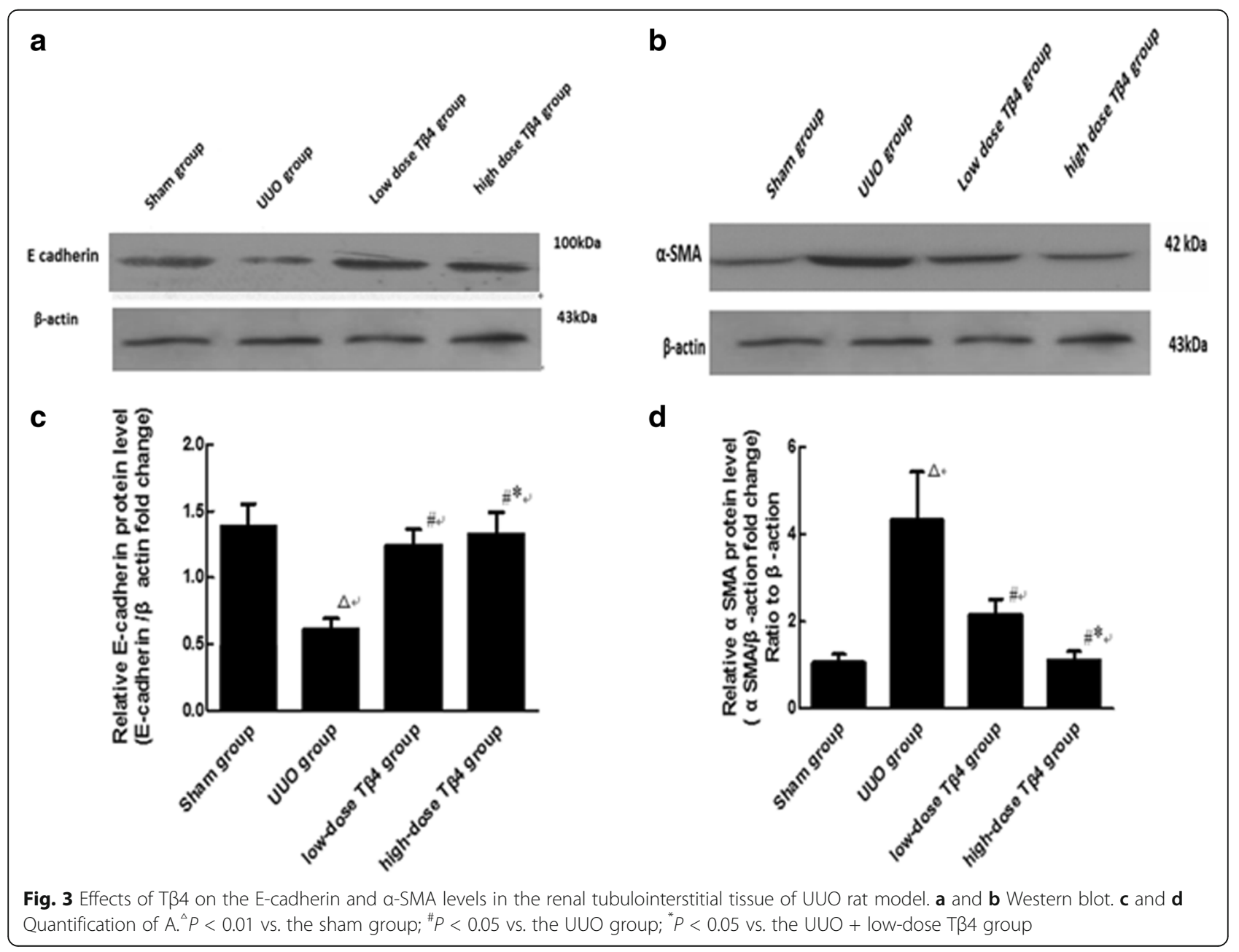


significantly elevated compared to the sham group (Fig. 3b, d) $(P<0.01)$. The expression of $\alpha$-SMA in the UUO + low-dose group was markedly decreased $(P<0.05)$. The reduction in $\alpha$-SMA expression was greater in the $\mathrm{UUO}+$ high-dose group compared to the UUO + low-dose group (Fig. 3b, d) $(P<0.05)$.

\section{Effects of different doses of T $\beta 4$ on E-cadherin and a-SMA} in TGF- $\beta$ induced renal tubular cells

$E$-cadherin mRNA in the TGF- $\beta$ group was significantly lower compared to the control group (Fig. $4 \mathrm{a}, \mathrm{P}<0.01$ ), the expression of E-cadherin mRNA in the TGF- $\beta+$ low-dose $\mathrm{T} \beta 4$ group was markedly increased compared to the TGF$\beta$ group (Fig. $4 \mathrm{a}, \mathrm{P}<0.05$ ). The increase in E-cadherin
mRNA expression was greater in the TGF- $\beta+$ high-dose $\mathrm{T} \beta 4$ group compared to the low-dose group (Fig. 4a, $\mathrm{P}<0.05)$. $\quad$-SMA mRNA expression in the TGF- $\beta$ group was significantly elevated compared to the control group (Fig. 4b $P<0.01$ ). The expression of $\alpha$-SMA mRNA in the TGF- $\beta+$ low-dose T $\beta 4$ group was markedly decreased (Fig. 4b $P<0.05)$. The reduction in $\alpha$-SMA expression was greater in the TGF- $\beta$ + high-dose T $\beta 4$ group compared to the low-dose group (Fig. $4 \mathrm{~b} P<0.05$ ).

E-cadherin expression in the TGF- $\beta$ group was significantly lower compared to the control group (Fig. 4c, d) $(P<0.01)$. The expression of E-cadherin in the TGF$\beta+$ low-dose $\mathrm{T} \beta 4$ group was markedly increased compared to the TGF- $\beta$ group $(P<0.05)$. The increase in E-cadherin

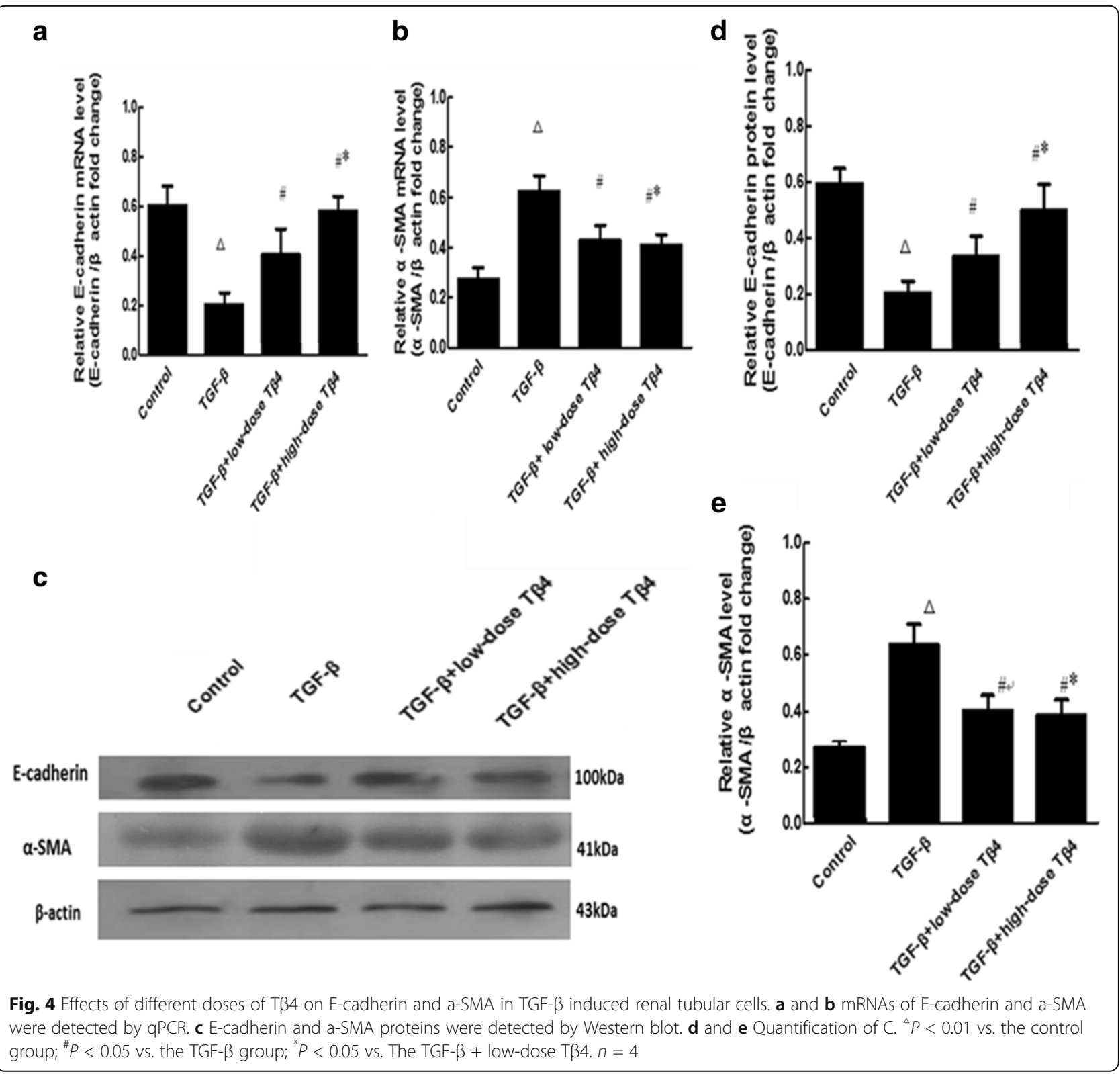


expression was greater in the TGF- $\beta+$ high-dose T $\beta 4$ group compared to the low-dose group (Fig. 4c, d) $(P<0.05)$. $\alpha$-SMA expression in the TGF- $\beta$ group was significantly elevated compared to the control group (Fig. 4c, e) $(P<0.01)$. The expression of $\alpha$-SMA in the TGF- $\beta$ + low-dose T $\beta 4$ group was markedly decreased $(P<0.05)$. The reduction in $\alpha$-SMA expression was greater in the TGF- $\beta$ + high-dose T $\beta 4$ group compared to the low-dose group (Fig. 4c, e) $(P<0.05)$.

\section{Effects of different doses of $T \beta 4$ on renal tubular cells} apoptosis in the UUO rat models

TUNEL staining of kidney sections in the UUO group was significantly strengthened compared to the sham group (Fig. 5a, b) $(P<0.01)$. The TUNEL staining in the low-dose T $\beta 4$ group was markedly weakened $(P<0.05)$. The reduction in TUNEL staining was greater in the high-dose T $\beta 4$ group compared to the low-dose group (Fig. 5a, b) $(P<0.05)$.

Significantly decreased Bcl-2 in the UUO group compared with sham group was found (Fig. 5c, d) $(P<0.01)$.
The expression of $\mathrm{Bcl}-2$ in the low-dose $\mathrm{T} \beta 4$ group was markedly increased compared to the UUO group $(P<0.05)$. The increase in $\mathrm{Bcl}-2$ expression was greater in the high-dose $\mathrm{T} \beta 4$ group compared to the low-dose group (Fig. 5c, f) $(P<0.05)$. The expressions of Bax and cleaved caspase- 3 in the UUO group were significantly elevated compared to the sham group (Fig. 5c-e) $(P<0.01)$. Compared with UUO group, the expressions of Bax and cleaved caspase- 3 in the low-dose and highdose $\mathrm{T} \beta 4$ groups were markedly decreased $(P<0.05)$.

T $\beta 4$ treatment attenuated apoptosis induced by TGF- $\beta$ in renal tubular cells

Observations of morphological alterations of apoptosis cells were addressed by tunnel staining and counted by Image J software. The number of apoptosis cells in the TGF- $\beta$ group was significantly elevated compared to the control group (Fig. 6a, b $P<0.01$ ). The number of apoptosis cells in the TGF- $\beta+$ low-dose T $\beta 4$ group was markedly decreased (Fig. 6a, b $P<0.05$ ). The reduction in apoptosis cells was greater in the TGF- $\beta+$ high-dose

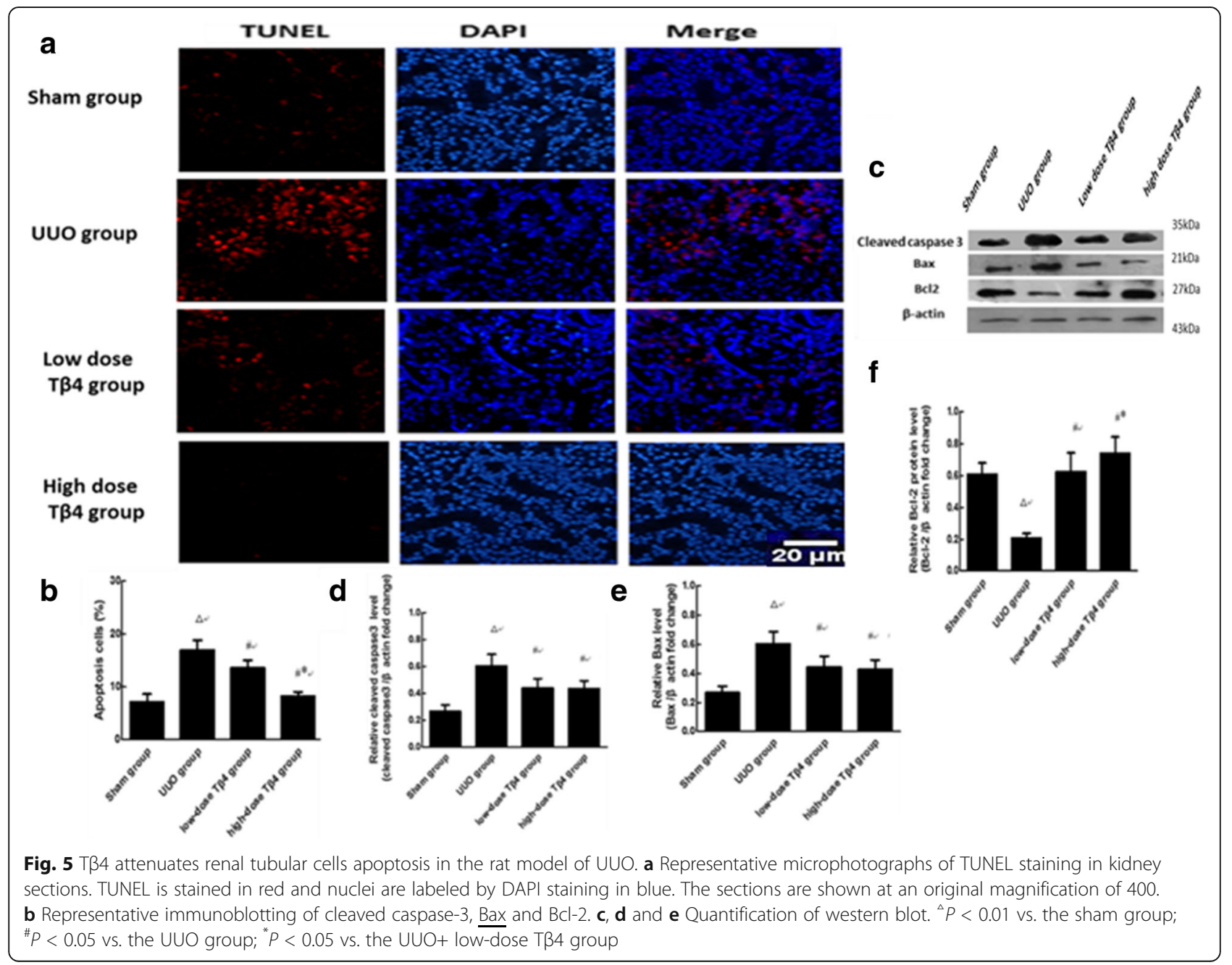




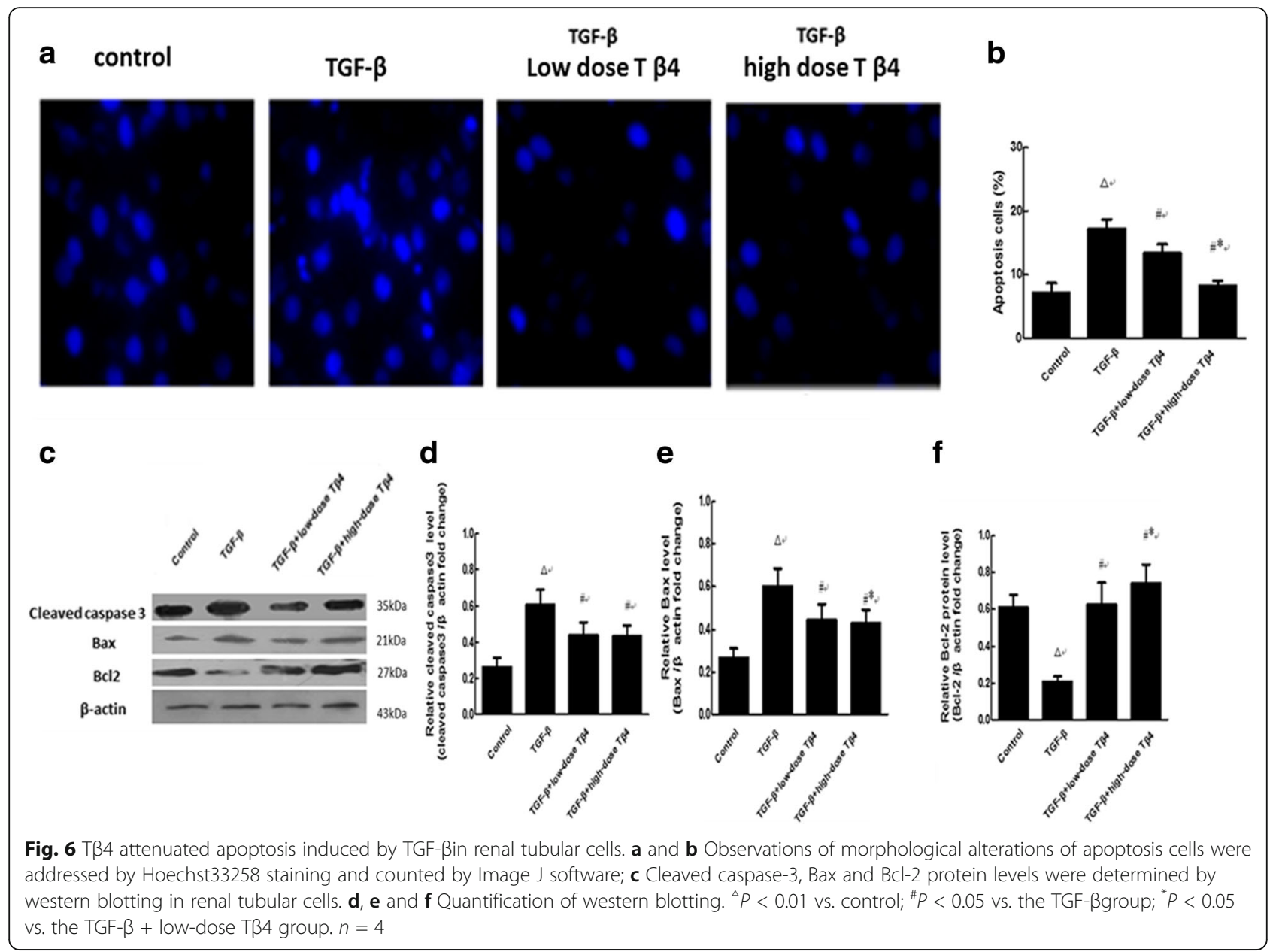

T $\beta 4$ group compared to the low-dose group (Fig. 6a, b $P<0.05)$.

The cleaved caspase- 3 and Bax expressions in the TGF$\beta$ group were significantly elevated compared to the control group (Fig. $6 \mathrm{c}-\mathrm{e} P<0.01$ ). The expressions of cleaved caspase- 3 and Bax in the TGF- $\beta$ + low-dose or high-dose T $\beta 4$ group were markedly decreased (Fig. $6 c-e ~ P<0.05$ ). The reduction in Bax was greater in the TGF- $\beta+$ highdose T $\beta 4$ group compared to the low-dose group (Fig. 6c, e $P<0.05)$. Bcl- 2 expression in the TGF- $\beta$ group was significantly lower compared to the control group (Fig. 6c, f) $(P<0.01)$. The expression of Bcl-2 in the TGF- $\beta+$ lowdose $\mathrm{T} \beta 4$ group was markedly increased compared to the TGF- $\beta$ group $(P<0.05)$. The increase in $\mathrm{Bcl}-2$ expression was greater in the TGF- $\beta+$ high-dose T $\beta 4$ group compared to the low-dose group (Fig. 6c, f) $(P<0.05)$.

\section{Discussions}

Thymosin is a lymphokine produced by the thymus and was first isolated from calf thymic proteins by Goldstein and White in 1966. Thymosins are small polypeptides containing more than 40 components [4]. T $\beta 4$ has the widest distribution and accounts for $70-80 \%$ of total thymosins [17-19]. Currently, synthetic $\mathrm{T} \beta 4$ has been used in experiments to explore the mechanisms underlying physiological and pathological activities [20-22]. The inhibitory effects of $\mathrm{T} \beta 4$ on some fibrosis factors in rats with progressive chronic renal fibrosis have been examined [12, 13, 23], but few study reported the inhibitory effects of T $\beta 4$ on TGF- $\beta$. Therefore, this study aimed to examine the effects of different doses of $T \beta 4$ on CRTIF and on the expression of TGF- $\beta$, E-cadherin, and $\alpha$-SMA.

Results of this showed that two weeks after UUO, there were no differences in kidney function among the four groups. Compared to the UUO group, T $\beta 4$ treatment decreased the 24-h proteinuria, reduced the area of pathological change, and decreased the $\alpha$-SMA expression. Compared to the UUO group, lower levels of TGF- $\beta$ protein expression were observed in UUO rats when different doses of $\mathrm{T} \beta 4$ were used for treatment. The levels of E-cadherin protein were lower in the UUO group. The above changes were more obvious in the high-dose T $\beta 4$ group than in low- dose $\mathrm{T} \beta 4$ group. Cell 
apoptosis in the renal tissue was improved by $\mathrm{T} \beta 4$ treatment in vivo. The results of tubular epithelial cells stimulated by TGF- $\beta$ shows that $\alpha$-SMA mRNA and protein levels decreased, E-cadherin mRNA and protein levels increased through T $\beta 4$ treatment, and similarly, these changes were more significant in the TGF- $\beta+$ high-dose T $\beta 4$ group. The apoptosis of tubular epithelial cells was improved by $\mathrm{T} \beta 4$ treatment compared with pure TGF- $\beta$ stimulation, and equally, the decrease of apoptosis was more obvious in the TGF- $\beta+$ high-dose T $\beta 4$ group in vitro. These results suggest that $\mathrm{T} \beta 4$ treatment might alleviate the kidney fibrosis and apoptosis of tubular epithelial cells by TGF- $\beta$ pathway inhibition in rats with CKD.

Renal tubulointerstitial fibrosis is a common feature of CKD and a main determinant of progression of renal diseases $[4,12]$. The UUO model is an experimental model of renal disease for renal tubulointerstitial fibrosis, chronic inflammation, and interstitial fibrosis caused by continuous ureteral obstruction that can lead to progressive loss of renal function [16]. During the process of fibrosis, over-secretion of TGF- $\beta$ is regarded as a key factor promoting fibrosis [5]. TGF- $\beta$ also promotes the transformation of epithelial cells in the renal tubules and capsule cells into fibroblasts, reducing E-cadherin expression and upregulating $\alpha$-SMA expression [6]. TGF- $\beta$ can induce fibroblasts cells to express $\alpha$-SMA, increasing the shrinkage of the fibrosis lesions, consequently leading to ischemia [24]. In this study, the UUO group showed histopathological lesions characteristic of kidney fibrosis, as well as high expression of TGF- $\beta$ and $\alpha-$ SMA.E-cadherin is a transmembrane glycoprotein maintaining the polarity of epithelial cells and establishing tight junctions. Lower levels of E-cadherin result in the separation of the epithelial cells, changes of phenotypes, and/or cell apoptosis. Lower levels of E-cadherin also reflect the early changes observed during epithelialmesenchymal transition(EMT) $[25,26]$. In this study, Ecadherin expression was decreased in the UUO model group compared to the sham group, but $\mathrm{T} \beta 4$ treatment increased the expression of E-cadherin, suggesting decreased EMT and alleviation of kidney fibrosis. In this study, different doses of $\mathrm{T} \beta 4$ decreased alleviated kidney fibrosis, suggesting that T $\beta 4$ could antagonize the expression of TGF- $\beta$ and $\alpha$-SMA, inhibit cell apoptosis, and increase E-cadherin expression, so reduce EMT. Additional comprehensive studies will be necessary to confirm the mechanism.

\section{Conclusions}

In conclusion, this study suggests that $\mathrm{T} \beta 4$ treatment might alleviate the kidney fibrosis and apoptosis of tubular epithelial cells through TGF- $\beta$ pathway inhibition in rats with CRTIF.T $\beta 4$ could be a novel way to treat CKD, but additional studies are still necessary before clinical trials can be performed.

\section{Additional file}

Additional file 1: Figure S1. Effects of TB4 alone on the E-cadherin, aSMA, cleaved caspase 3,Bax and Bcl2 levels in the renal tubular cells. (A) Western blot.(B) Quantification of A. The results between control group and Tß4 treated group had no significant difference. (PNG $40 \mathrm{~kb}$ )

\section{Funding}

This study was supported by Guizhou province international technology cooperation plan(20137028), Guizhou provincial fine-quality green branch specialized talents cultivation fund(201343), The national natural science foundation of China (81200539), Guizhou province department of a joint fund(20147002).

\section{Authors' contributions \\ $J Y$ and $Y Z$ carried out the studies, participated in collecting data, and drafted the manuscript. $X Y, Y S$ and $Y X$ performed the statistical analysis and participated in its design. $X \mathrm{~L}, \mathrm{WZ}$ and MT, $Y Z$ helped to draft the manuscript. All authors read and approved the final manuscript.}

\section{Competing interest}

The authors declare that they have no competing interest.

\section{Ethics approval and consent to participate}

This study was approved by the Experimental Ethics Committee of Guizhou Medical University. Consent was not required.

Consent for publication

Not applicable.

\section{Publisher's Note}

Springer Nature remains neutral with regard to jurisdictional claims in published maps and institutional affiliations.

Received: 11 December 2015 Accepted: 24 August 2017

Published online: 18 October 2017

References

1. Kidney Disease: Improving Global Outcomes (KDIGO) CKD Work Group. KDIGO 2012 clinical practice guideline for the evaluation and management of chronic kidney disease. Kidney Int Suppl. 2013;3:4.

2. Zhang L, Wang F, Wang L, Wang W, Liu B, et al. Prevalence of chronic kidney disease in China: a cross-sectional survey. Lancet. 2012;379:815-22.

3. Liu Y. Renal fibrosis: new insights into the pathogenesis and therapeutics. Kidney Int. 2006;69:213-7.

4. Boor P, Ostendorf T, Floege J. Renal fibrosis: novel insights into mechanisms and therapeutic targets. Nat Rev Nephrol. 2010;6:643-56.

5. Qiao X, Li RS, Li H, Zhu GZ, Huang XG, Shao S, et al. Intermedin protects against renal ischemia-reperfusion injury by inhibition of oxidative stress. Am J Physiol Renal Physiol. 2013;304:F112-9.

6. Yang J, Liu Y. Dissection of key events in tubular epithelial to myofibroblast transition and its implications in renal interstitial fibrosis. Am J Pathol. 2001; 159:1465-75.

7. Wight TN, Potter-Perigo S. The extracellular matrix: an active or passive player in fibrosis? Am J Physiol Gastrointest Liver Physiol. 2011;301:G950-5.

8. Goldstein AL, Slater FD, White A. Preparation, assay, and partial purification of a thymic lymphocytopoietic factor (thymosin). Proc Natl Acad Sci U S A. 1966:56:1010-7.

9. Crockford D, Turjman N, Allan C, Angel J. Thymosin beta4: structure, function, and biological properties supporting current and future clinical applications. Ann N Y Acad Sci. 2010;1194:179-89.

10. Goldstein AL, Hannappel E, Kleinman HK. Thymosin beta4: actin-sequestering protein moonlights to repair injured tissues. Trends Mol Med. 2005;11:421-9.

11. Pai M, Spalding D, Xi F, Habib N. Autologous bone marrow stem cells in the treatment of chronic liver disease. Int J Hepatol. 2012;2012:307165. 
12. Liu C, Mei W, Tang J, Yuan Q, Huang L, Lu M, et al. Mefunidone attenuates tubulointerstitial fibrosis in a rat model of unilateral ureteral obstruction. PLoS One. 2015;10:e0129283.

13. Zuo Y, Chun B, Potthoff SA, Kazi N, Brolin TJ, Orhan D, et al. Thymosin beta4 and its degradation product, ac-SDKP, are novel reparative factors in renal fibrosis. Kidney Int. 2013;84:1166-75.

14. Moriyama T, Kawada N, Ando A, Horio M, Yamauchi A, Na-gata K, et al. Up-regulation of HSP47 in the mouse kidneys with unilateral ureteral obstruction. Kidney Int. 1998;54:110-9.

15. Woessner JF Jr. The determination of hydroxyproline in tissue and protein samples containing small proportions of this imino acid. Arch Biochem Biophys. 1961;93:440-7.

16. Lee SM, Rao VM, Franklin WA, Schiffer MS, Aronson AJ, Spargo BH, et al. IgA nephropathy: morphologic predictors of progressive renal disease. Hum Pathol. 1982:13:314-22.

17. Koutrafouri V, Leondiadis L, Avgoustakis K, Livaniou E, Czarnecki J, Ithakissios DS, et al. Effect of thymosin peptides on the chick chorioallantoic membrane angiogenesis model. Biochim Biophys Acta. 2001;1568:60-6.

18. Kumar S, Gupta S. Thymosin beta 4 prevents oxidative stress by targeting antioxidant and anti-apoptotic genes in cardiac fibroblasts. PLoS One. 2011; 6:e26912.

19. Ock MS, Cha HJ, Choi YH. Verifiable hypotheses for thymosin beta4-dependent and -independent angiogenic induction of Trichinella spiralis-triggered nurse cell formation. Int J Mol Sci. 2013;14:23492-8.

20. Xiao Y, Qu C, Ge W, Wang B, Wu J, Xu L, et al. Depletion of thymosin beta4 promotes the proliferation, migration, and activation of human hepatic stellate cells. Cell Physiol Biochem. 2014;34:356-67.

21. Kim J, Wang S, Hyun J, Choi SS, Cha H, Ock M, et al. Hepatic stellate cells express thymosin Beta 4 in chronically damaged liver. PLoS One. 2015;10: e0122758.

22. Qiu P, Wheater MK, Qiu Y, Sosne G. Thymosin beta4 inhibits TNF-alphainduced NF-kappaB activation, IL-8 expression, and the sensitizing effects by its partners PINCH-1 and ILK. FASEB J. 2011:25:1815-26.

23. Goldstein AL, Hannappel E, Sosne G, Kleinman HK. Thymosin beta4: a multi-functional regenerative peptide. Basic properties and clinical applications. Expert Opin Biol Ther. 2012;12:37-51.

24. Gagliardini $\mathrm{E}$, Benigni $\mathrm{A}$. Therapeutic potential of TGF-beta inhibition in chronic renal failure. Expert Opin Biol Ther. 2007;7:293-304.

25. Tunggal JA, Helfrich I, Schmitz A, Schwarz H, Gunzel D, Fromm M, et al. E-cadherin is essential for in vivo epidermal barrier function by regulating tight junctions. EMBO J. 2005;24:1146-56.

26. Liu Y. New insights into epithelial-mesenchymal transition in kidney fibrosis. J Am Soc Nephrol. 2010;21:212-22.

\section{Submit your next manuscript to BioMed Central and we will help you at every step:}

- We accept pre-submission inquiries

- Our selector tool helps you to find the most relevant journal

- We provide round the clock customer support

- Convenient online submission

- Thorough peer review

- Inclusion in PubMed and all major indexing services

- Maximum visibility for your research

Submit your manuscript at www.biomedcentral.com/submit 